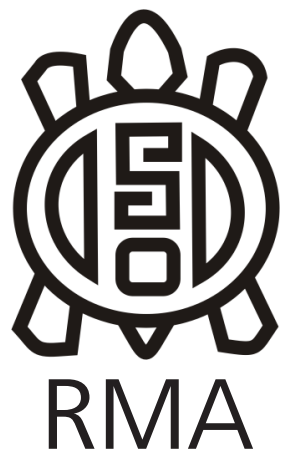

Dossier

\title{
Arqueología experimental para el análisis lítico: algunos ejemplos de aplicación en arqueología argentina
}

\author{
Experimental archaeology for lithic analysis: some examples of \\ application in Argentine archaeology \\ Celeste Weitzel*, Damián Bozzuto** y Hernán De Angelis*** \\ * CONICET, Área Arqueología y Antropología, Museo de Ciencias Naturales, Necochea, Argentina. \\ E-mail: celweitzel@gmail.com \\ ** Instituto Nacional de Antropología y Pensamiento Latinoamericano-CONICET-Universidad de \\ Buenos Aires, Buenos Aires, Argentina. E-mail: dbozzuto@filo.uba.ar \\ ${ }^{* * *}$ Centro Austral de Investigaciones Científicas-CONICET, Ushuaia, Argentina. \\ E-mail: hernandeangelis@yahoo.com.ar
}

El Primer Congreso Argentino de Estudios Líticos en Arqueología (CAELA), tuvo lugar en septiembre de 2018 en la ciudad de Córdoba. Este encuentro presentó la oportunidad para debatir y actualizar las diversas investigaciones desarrolladas en torno a los análisis líticos en el ámbito nacional. En este marco, se llevó a cabo el simposio de Arqueología Experimental del cual surgen los trabajos reunidos en este volumen especial.

La Arqueología Experimental implica la simulación o la replicación de objetos, técnicas, tecnologías, sistemas o situaciones, mediante distintos tipos de experimentos y experiencias. Siempre sobre la base de la evidencia arqueológica, la finalidad es estudiar y ofrecer elementos para la interpretación de la materialidad de las sociedades pasadas (Amick, Mauldin y Binford, 1989; Coles, 1979; O'Sullivan et al., 2014). La experimentación permite observar de manera directa relaciones de causa-efecto y realizar inferencias sobre prácticas, comportamientos y situaciones que pudieron suceder en el pasado pero que hoy no pueden ser observados (Ascher, 1961; Nami, 1991). De esta manera, la Arqueología Experimental sirve para generar y contrastar hipótesis sobre el pasado a través de la analogía experimental (Ascher 1961; Amick et al., 1989; Eren et al., 2016.; Nami, 2018). Asimismo, más recientemente crece su utilización como recurso didáctico educativo y de comunicación pública de la ciencia (Eren, 2009; Peterssen \& Narmo, 2011).

Si bien la Arqueología Experimental es una forma de generar información, herramientas y preguntas para la interpretación del registro arqueológico, implica forzosamente una simplificación de la realidad a observar. Los experimentos arqueológicos se han caracterizado usualmente en función del grado de control de las variables y el ámbito en el que se realizan. Aunque muchos investigadores han propuesto clasificaciones particulares de los tipos de experimentos, en líneas generales se podrían separar en dos conjuntos. Por un lado, las experiencias que se desarrollan en "condiciones de laboratorio", bajo un riguroso control de las variables seleccionadas, que implica observaciones inducidas de forma artificial con el uso de tecnología adecuada para poder reproducir y medir lo observado (Schenck, 2011; Reynolds, 1999; Tringham, 1978). Por otro lado, los llamados experimentos integrales, simulativos o actualísticos, que implican la simulación y/o replicación experimental de actividades, procesos, condiciones materiales, técnicas etc. que pudieron tener lugar en el pasado, observando las posibles relaciones causa-efecto que no pueden observarse en el registro, y contrastando los resultados con el registro arqueológico (Ascher 1961, Outram 2008). Este proceso de observación, experimentación e interpretación usualmente lleva a generar nuevas inferencias y preguntas, así como eventualmente al perfeccionamiento de nuestras hipótesis e interpretaciones (Amick et al., 1989; Ascher, 1961; Outram, 2008). La elección de una u otra forma de experimentación se asocia a los problemas y preguntas a resolver, pero también muchas veces va de la mano de un posicionamiento teórico/conceptual. Sin embargo, los distintos enfoques experimentales no son excluyentes, sino que pueden complementarse el uno al otro (Outram, 2008).

La tecnología lítica, ha brindado un vasto campo de aplicación para los estudios experimentales (i.e. Eren et al., 2016; Nami, 2018). Este tipo de acercamiento resulta de gran ayuda cuando nos enfrentamos a nuevos problemas, como por ejemplo identificar la utilización de distintas técnicas de talla, inferir secuencias de producción de objetos específicos o dentro del conjunto lítico de un sitio, identificar tratamientos térmicos, enmangues, uso o tipos de alteraciones resultado de diferentes procesos tafonómicos y/o post-depositacionales. También ha servido como base para la construcción de modelos y para evaluar la efectividad de la aplicación de distintas metodologías de análisis lítico (Eren et al., 2016; Nami, 1982, 2018).

En el simposio de Arqueología Experimental del $1{ }^{\circ} \mathrm{CAELA}$, se trató de reunir un panorama de trabajos experimentales para el análisis de los materiales líticos que se están realizando en nuestro país, presentando las diferentes metodologías y herramientas que se vienen desarrollando y aplicando para ello. Los trabajos reunidos a continuación fueron ordenados siguiendo los distintos momentos de la historia de vida (Hurcombe, 2007) de los objetos líticos, cubriendo desde el momento de talla y producción de artefactos, los estudios del uso, incluyendo su participación en la confección de otras tecnologías, y estudios de los procesos ocurridos durante la depositación de los artefactos líticos o luego de esta. Los experimentos presentados tienen generalmente un mayor énfasis en la perspectiva simulativa, con diversos grados de control de variables y de uso de instrumental de laboratorio.

En el primer artículo "Experimentos y esquemas diacríticos para explorar técnicas de talla unifacial del Holoceno temprano en el noroeste de Santa Cruz", Teresa Civalero y Hugo Nami presentan un experimento replicativo diseñado para conocer la cadena de producción asociada con una 
modalidad particular de confección de instrumentos unifaciales durante el Holoceno temprano. Esta experimentación se utiliza para obtener información de base para la lectura diacrítica de los materiales replicados, que luego servirá para el análisis de los instrumentos unifaciales arqueológicos. Los resultados señalan una intencionalidad en el modo de confección de estos artefactos y la posible existencia de un estilo técnico compartido durante el Holoceno temprano en la Patagonia centro-meridional.

El segundo artículo "El uso del cuarzo en la Sierra El AltoAncasti (Catamarca, Argentina). Experimentación y casos arqueológicos" es de autoría de Débora Egea e Ignacio Gerola. Se presenta la experimentación con distintas técnicas de talla aplicadas al cuarzo, para obtener datos para el análisis de la tecnología lítica de la Sierra de El AltoAncasti. Los autores utilizan la talla directa y la bipolar para la confección de artefactos experimentales que les permitan evaluar los rasgos diagnósticos de la talla del cuarzo, particularmente en las primeras etapas de producción lítica.

El artículo de Marcela Leipus y Eduardo Apolinaire se titula "Análisis funcional de base microscópica aplicado a materias primas de la provincia de Entre Ríos: identificación y caracterización de rastros de uso experimentales". Este trabajo presenta un programa experimental para la caracterización de rastros de uso sobre diversas materias primas que han sido poco exploradas desde la perspectiva funcional. Estas rocas afloran en las cuencas de los ríos Paraná y Uruguay y se encuentran representadas en diversos conjuntos líticos del nordeste argentino. A partir de este experimento, los autores logran identificar y caracterizar los rastros de uso generados en estas rocas mediante el trabajo sobre diversos materiales con diferentes cinemáticas, conformando un marco de referencia sólido para el posterior estudio de los artefactos arqueológicos a nivel regional

El siguiente trabajo, realizado por Erico Gáal y Andrés Chesini Remic, lleva por título "Trabajo experimental en metales mediante el uso de artefactos líticos. Posibles implicancias para el taller metalúrgico de Rincón Chico 15 (sur del valle de Yocavil, prov. de Catamarca)". En este caso se utiliza la experimentación para realizar una primera aproximación al problema de la funcionalidad que pudieron tener los artefactos líticos en la producción de objetos de oro y cobre. Por un lado, los autores replicaron los artefactos líticos hallados en el sitio Rincón Chico 15, que utilizaron para reproducir posibles técnicas de trabajo de metales. Luego, realizaron el análisis funcional de base microscópica de los artefactos experimentales y arqueológicos para elaborar una colección de referencia y una base de datos asociando determinadas formas, usos potenciales y rastros microscópicos.

Finalmente, Ariel Frank y Juan Baridón presentan una experimentación destinada a evaluar la distribución de los restos líticos que son descartados en estructuras de combustión, en su artículo "Las piedras al fuego. Experimentos para abordar la distribución espacial de restos líticos termoalterados". Los autores utilizan lascas experimentales de distintas materias primas que son arrojadas al fuego hasta que finaliza la combustión de los fogones y luego analizan los patrones de distribución de los fragmentos líticos. Sus resultados señalan una relación entre estos patrones y el tipo de materia prima y el tamaño de las piezas expuestas al fuego, lo que les permite postular expectativas arqueológicas para diversos contextos de artefactos líticos caídos al fuego.

\section{Referencias citadas}

Ascher, R. (1961). Experimental Archaeology. American Anthropologist, 63(4), 763-816.

Coles, J. (1979). Experimental Archaeology. Londres: London Academic Press.

Eren, M., Lycett, S., Patten, R., Buchanan, B., Pargeter, J., \&. O'Brien, M.J. (2016). Test, Model, and Method Validation: The Role of Experimental Stone Artifact Replication in Hypothesis-driven Archaeology, Ethnoarchaeology, 8(2), 103-136. DOI: 10.1080/19442890.2016.1213972.

Eren, M. (2009). Experimental archaeology as a pillar of archaeological education. Nikolay Arkeologisk Tidsskrift, 107(1), 25-32.

Hurcombe, L. M. (2007) Archaeological Artefacts as Material Culture. Londres, New York: Routtledge.

Nami, H. (1982). La Arqueología Experimental. Enfoque Antropológico, 1(1), 1-7.

Nami, H. (1991). Algunas reflexiones teóricas sobre arqueología y experimentación. Shincal, 3(1), 151-168.

Nami, H. (2018). Theoretical and Epistemological Thoughts on Archaeology and Experimental Lithic Technology. Journal of Research in Philosophy and History, 1(2), 139-165.

Outram, A. (2008). Introduction to experimental archaeology. World Archaeology, 40(1), 1-6. DOI: 10.1080/00438240801889456

O'Sullivan, A., Powers, M., Murphy, J., Inwood, N., Gilhooly, B., Kelly, N., Malone, W., Mulrooney, J., Corrigan, C., L'Estrange, M., Burke, A., Kazuro, M., McDermott, C., Warren, G., O'Neill, B., Heffernan M., \& Sweeney, M. (2009). Experimental archaeology: making; understanding; story-telling. En Kelly, B., Roycroft, N., \& Stanley, M. (Eds.), Fragments of Lives Past: archaeological objects from Irish road schemes (pp. 115-126). Dublin: National Roads Authority.

Petersson, B., \& Narmo, L. (Eds.), Experimental Archaeology, Between Enlightenment and Experience. Lund: Lund University.

Reynolds, P. J. (1999). The nature of experiment in archaeology. En Harding, A. F. (Ed.), Experiment and Design: Archaeological Studies in Honour of John Coles (pp. 156-162). Oxford: Oxbow Books.

Schenck, T. (2011). Experimenting with the Unknown. En Petersson, B. \& Narmo, L. (Eds.), Experimental Archaeology, Between Enlightenment and Experience (pp. 87-98). Lund: Lund University.

Tringham, R. (1978). Experimentation, Ethnoarchaeology and the Leapfrogs in Archaeological Methodology. En Gould, R. (Ed.), Explorations in Ethnoarchaeology (pp.169-200). Albuquerque: University of New Mexico Press. 